\title{
Heavy-Atom Effect Promotes Multi-Resonance Thermally Activated Delayed
}

\section{Fluorescence}

Tao Hua,${ }^{a, b,+}$ Lisi Zhan, ${ }^{c,+}$ Nengquan Li, ${ }^{a}$ Zhongyan Huang, ${ }^{a, *}$ Xiaosong Cao, ${ }^{a}$ Zhengqi

Xiao, ${ }^{a}$ Shaolong Gong, ${ }^{c, *}$ Changjiang Zhou, ${ }^{a}$ Cheng Zhong, ${ }^{c}$ Chuluo Yang ${ }^{a, c, *}$

${ }^{a}$ Shenzhen Key Laboratory of Polymer Science and Technology, College of Materials

Science and Engineering, Shenzhen University, Shenzhen 518060, People's Republic of China

E-mail: zyhuang@szu.edu.cn (Z.H.); clyang@ szu.edu.cn (C.Y.)

${ }^{b}$ College of Physical and Optoelectronic Engineering, Shenzhen University, Shenzhen 518060, People's Republic of China

${ }^{c}$ Hubei Key Lab on Organic and Polymeric Optoelectronic Materials, Department of Chemistry, Wuhan University, Wuhan, 430072, People's Republic of China

Email: slgong@whu.edu.cn (S.G.)

${ }^{+}$These authors contributed equally to this work. 


\section{Abstract}

As one type of latest emitters with simultaneous high efficiency and color-purity, the development of multi-resonance thermally activated delayed fluorescence (MR-TADF) materials represents an important advancement for organic light-emitting diodes (OLEDs). We herein present a new strategy to improve the performance of MR-TADF emitters by fusing sulfur element into the B-N based framework, aiming to utilize the non-metal heavy-atom effect in accelerating the reverse intersystem crossing (RISC) process of the emitter. Two compounds, namely 2PTZBN and 2PXZBN, were developed in this work through rigidifying the DABNA-1 skeleton by sulfur or oxygen atoms. The theoretical calculations and photoluminescence studies revealed that the sulfur-incorporated 2PTZBN enabled considerable rate constant of RISC ( $\left.k_{\mathrm{RISC}}\right)$ up to $2.8 \times 10^{5} \mathrm{~s}^{-1}$ in toluene due to larger spin-orbital coupling (SOC) values and smaller singlet-triplet energy splitting $\left(\Delta E_{\mathrm{ST}}\right)$ compared with 2PXZBN. Consequently, organic light-emitting diodes based on 2PTZBN exhibited highly efficient green emission with maximum external quantum efficiency (EQE) of $25.5 \%$.

Keywords: multi-resonance $\bullet$ thermally activated delayed fluorescence $\bullet$ heavy-atom effect• reverse intersystem crossing $\bullet$ organic light-emitting diodes 
Recently, a new concept termed multi-resonance (MR) effect has been adopted for pure organic thermally activated delayed fluorescence (TADF) emitters, leading to high-efficiency and color-purity organic light-emitting diodes (OLEDs). ${ }^{1-7}$ Boronbased MR-TADF emitters utilize the opposite resonance effects of the boron and nitrogen atoms in the core structure to realize efficient separation of the highest occupied molecular orbital (HOMO) and the lowest unoccupied molecular orbital (LUMO), providing small singlet-triplet energy splitting $\left(\Delta E_{\mathrm{ST}}\right)$ to facilitate harvesting of triplet excitons. ${ }^{1}$ In addition, the rigid nature of these materials can significantly reduce the vibrational motion to obtain narrow-band emission with very small fullwidth at half-maximum (FWHM) as well as large oscillator strength $(f)$, which can effectively address the predicaments in traditional donor-acceptor (D-A) type TADF emitters such as poor color purity and unsatisfactory quantum efficiency $\left(\Phi_{\mathrm{PL}}\right)^{6}$ Nonetheless, documented MR-TADF examples featuring promising electroluminescence remain sparse, and tactics to improve their efficiency are of great importance. $^{8-22}$

Reverse intersystem crossing (RISC) process is a critical pathway for emitters to achieve TADF. ${ }^{23-27}$ A fast rate constant of RISC ( $k_{\mathrm{RISC}}$ ) of TADF emitter can efficient converted its $T_{1}$ excitons to $S_{1}$ state, promising high internal quantum efficiency (IQE), while inefficient RISC would conversely increase the concentration of triplet excitons to induce triplet-triplet annihilation (TTA) or other degradation events. ${ }^{28}$ For MRTADF emitters, several strategies were introduced to enhance the $k_{\mathrm{RISCS}}$ and improve the efficiencies. ${ }^{1,17,29-34}$ For instance, Hatakeyama et al. reported that the introduction 
of diphenylamino and phenyl groups into the well-known DABNA-1 molecule could significantly promote the $k_{\mathrm{RISC}}$, and provide external quantum efficiency (EQE) upwards $20 \%$ in OLEDs. ${ }^{1}$ Wang et al. demonstrated that incorporation an auxiliary donor into an MR skeleton produced MR-TADF with very high $k_{\text {RISC }}\left(\sim 1.0 \times 10^{6} \mathrm{~s}^{-1}\right)$ and EQE (27\%) in device. ${ }^{32}$

According to Fermi's golden rule, a fast $k_{\text {RISC }}$ could be obtained by combining sufficient spin-orbit coupling (SOC) and tiny $\Delta E_{\mathrm{ST} .}{ }^{35}$ Albeit small $\Delta E_{\mathrm{ST}} \mathrm{S}(<0.2 \mathrm{eV})$ could be realized through a reasonable design of the resonance position between the electron deficient and electron donating units of MR-TADF materials, little attention has been paid to enlarge the SOC, which is essential and can be promoted by heavy elements. ${ }^{36-43}$ In this work, aiming to increase the SOC, sulfur (S) as heavy element was incorporated into the B-N fused polycyclic aromatic framework, and a novel MRTADF emitter, namely 2PTZBN, was designed and successfully synthesized by incorporating phenothiazine (PTZ) units into the B-N polycyclic framework. Meanwhile, the oxygen (O) doped counterpart, namely 2PXZBN, was also synthesized for comparison (Figure 1). The photoluminescence (PL) spectra of 2PXZBN and 2PTZBN bathochromic shifted to green region and showed similar narrow band emission with FWHMs less than $40 \mathrm{~nm}$ in solution state. The theoretical calculation of SOC and transient PL measurement of the emitters disclosed that the heavy atom effect (HAE) induced by S atom lead to larger SOC value, shorter DF lifetime and faster $k_{\mathrm{RISC}}$ value of $2 \mathrm{PTZBN}$ over $2 \mathrm{PXZBN}$. As a result, significant differences were found in device performance between the two emitters. The device based on 2PTZBN emitter 
exhibited high EQEs up to $25.5 \%$, which was significantly higher than $17.7 \%$ obtained from 2PXZBN emitter.
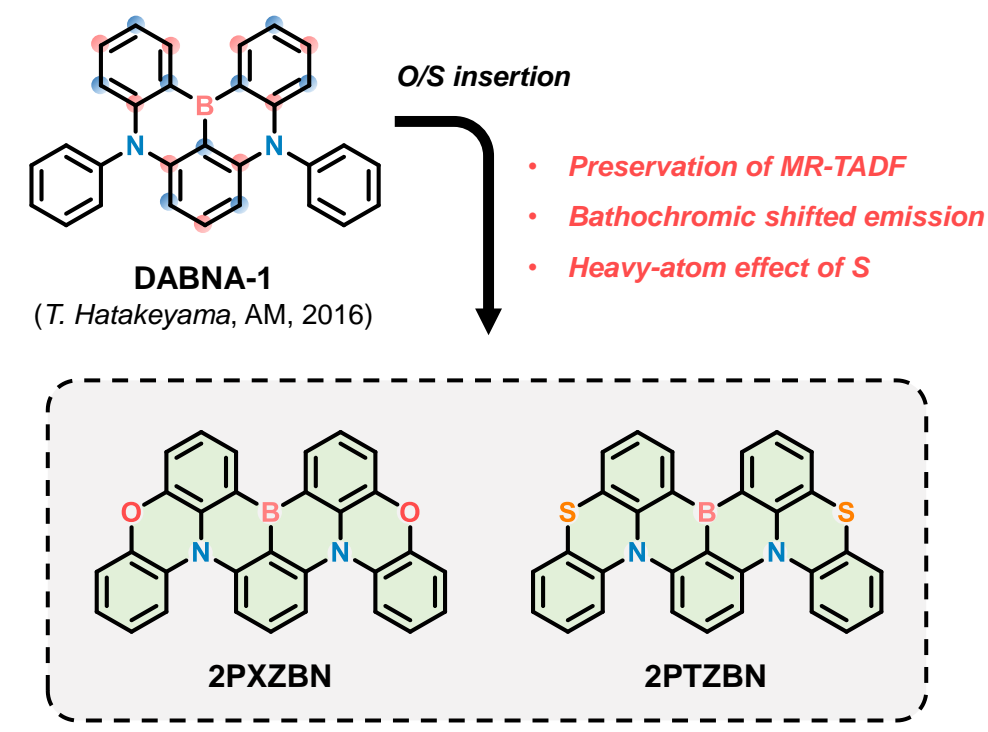

This work

Figure 1. The molecular design strategy and chemical structures of the MR-TADF emitters in this work.

2PXZBN and 2PTZBN were derived from the molecular structure of DABNA-1 by rigidifying the peripheral phenyl groups by $\mathrm{S}$ or $\mathrm{O}$ bridge (Figure 1). To investigate their electronic properties, density functional theory (DFT) and time-dependent DFT (TD-DFT) calculations were performed, and the results were presented in Figure 2. The optimized ground-state configurations revealed the twisted molecular structures for both compounds, where 2PTZBN exhibited larger dihedral angles between the planar of $\mathrm{B}-\mathrm{N}$ (A ring) and the tilted phenyl units ( $\mathrm{B}$ or B' ring), e.g., $28.1^{\circ}$ for $2 \mathrm{PXZBN}$ and $33.1^{\circ}$ for $2 \mathrm{PTZBN}$, induced by the larger radius of $\mathrm{S}$ atom compared with $\mathrm{O}$. The LUMOs of both new emitters were mainly localized on the boron atom and its 
ortho/para positions in triphenyl boron core, while the HOMOs dispersed on several parts including the nitrogen atoms, the meta positions of boron atom, the oxygen or sulfur atoms, and the tilted phenyl units outside the triphenyl boron skeleton. For 2PXZBN and 2PTZBN, the HOMO and LUMO were substantially separated while maintaining a certain degree of overlap, which may concurrently benefit a small $\Delta E_{\mathrm{ST}}$ and a large $f$ for the $\mathrm{S}_{1} \rightarrow \mathrm{S}_{0}$ transition. Compared with DABNA-1, ${ }^{1}$ PXZBN and 2PTZBN showed more delocalized HOMO and LUMO distribution due to extended conjugation, resulting in narrower bandgaps of $3.01 \mathrm{eV}$ for $2 \mathrm{PXZBN}$ and $3.31 \mathrm{eV}$ for 2PTZBN, with respect to $3.66 \mathrm{eV}$ for DABNA-1. In addition, the $f$ values of 2PXZBN and 2PTZBN are calculated to 0.2376 and 0.2285 , respectively, which are larger than that of DABNA-1 (0.2046) and could contribute to a higher singlet radiative decay rate. The SOC constants for $S_{1}-T_{1}, S_{1}-T_{2}$ and $S_{1}-T_{3}$ were then calculated and analyzed for DABNA-1, 2PXZBN, and 2PTZBN (Figure 2c). It was found that the SOC constants of $S_{1}-T_{1}$ were quite small for all the three emitters, indicating RISC process directly via $T_{1}-S_{1}$ channel should be slow. However, their SOC constants of $S_{1}-T_{2}$ and $S_{1}-T_{3}$ were significantly larger. For example, the SOC constants of $S_{1}-T_{1}, S_{1}-T_{2}$ and $S_{1}-T_{3}$ were calculated as $0.014,1.524$ and $1.187 \mathrm{~cm}^{-1}$, respectively for 2PTZBN, suggesting that $S_{1}-T_{2}$ or $S_{1}-T_{3}$ should be more efficient channels for energy up-conversion. These results indicate that the RISC from $T_{1}$ to $S_{1}$ in these emitters could be a higher-ordered process, which involves the $\mathrm{T}_{1}$ to $\mathrm{T}_{2}$ (or $\mathrm{T}_{3}$ ) reverse interconversion (RIC) followed by the $T_{2}$ (or $T_{3}$ ) to $S_{1}$ spin flip. ${ }^{35}$ It should also be noted that the SOC constants of $S_{1}-T_{2}$ exhibited a gradual increase in the order of DABNA-1 $\left(1.009 \mathrm{~cm}^{-1}\right), 2$ PXZBN $(1.118$ 
$\left.\mathrm{cm}^{-1}\right)$, and 2PTZBN $\left(1.524 \mathrm{~cm}^{-1}\right)$. The substantial difference in the case of 2PXZBN and $2 \mathrm{PTZBN}$ can be attributed to the existence of HAE induced by $\mathrm{S}$ atoms. It is anticipated that the larger SOC constant of $\mathrm{S}_{1}-\mathrm{T}_{2}$ channel in $2 \mathrm{PTZBN}$ will eventually translate into high $k_{\text {RISC. }}$

(a)

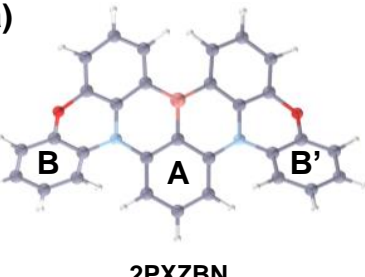

2PXZBN

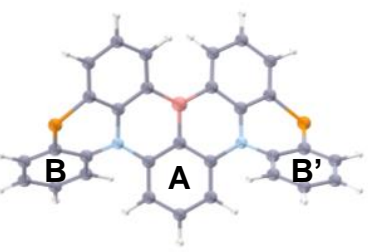

2PTZBN

(c)

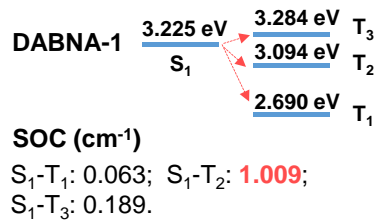

(b)

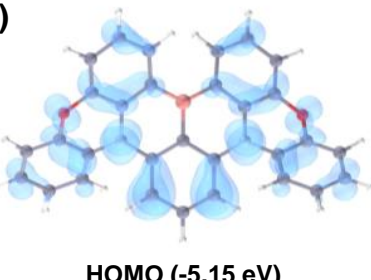

$f=0.2376$

$E_{\mathrm{g}}=3.01 \mathrm{eV}$
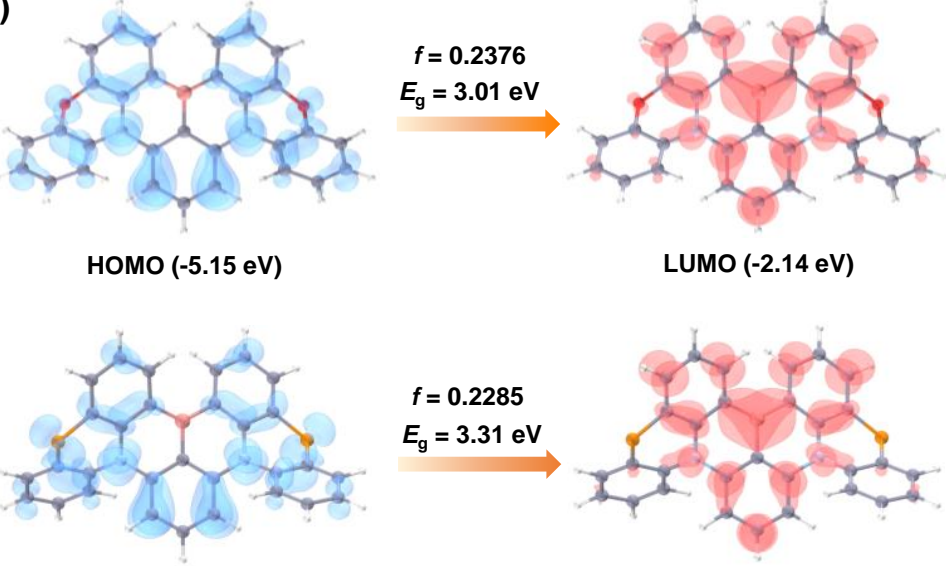

HOMO (-5.03 eV)

0.2285

$E_{\mathrm{g}}=3.31 \mathrm{eV}$

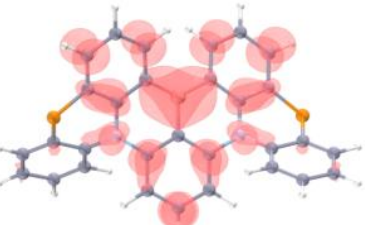

LUMO (-1.72 eV)

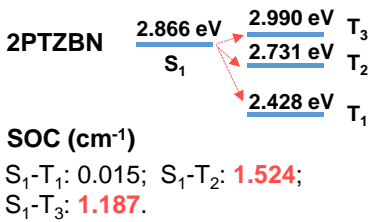

Figure 2. (a) Simulated structures of $2 \mathrm{PXZBN}$ and $2 \mathrm{PTZBN}$ in the ground states. (b) HOMO and LUMO distributions, energy band gaps, and oscillator strengths of 2PXZBN and 2PTZBN. (c) The SOC constants of DABNA-1, 2PXZBN and 2PTZBN.

2PXZBN and 2PTZBN were facilely synthesized through a Buchwald-Hartwig C-N coupling reaction and a one-pot borylation from the commercially available starting materials of phenoxazine (PXZ), phenothiazine (PTZ), and 1,3-dibromo-2chlorobenzene (see Supporting Information for details). The structures of the final products were carefully characterized by ${ }^{1} \mathrm{H}$ NMR, ${ }^{13} \mathrm{C}$ NMR, high resolution mass 
spectrometry (HR-MS), and further confirmed by single-crystal X-ray crystallographic analysis (Figure 3 and Table S1 and S2, Supporting Information). Thermogravimetric analysis (TGA) revealed the excellent thermal stability of the two compounds, with high decomposition temperatures ( $T_{\mathrm{d}}$, corresponding to $5 \mathrm{wt} \%$ loss) of $360{ }^{\circ} \mathrm{C}$ for 2PXZBN and $392{ }^{\circ} \mathrm{C}$ for $2 \mathrm{PTZBN}$ (Figure S5, Supporting Information), which will benefit the sublimation of the materials for purification and device fabrication and operation. The single-crystal structures clearly revealed larger twisted angles $\left(58.2^{\circ}\right)$ between the B-N planar (A ring) and the tilted phenyl units (B or B' ring) in 2PTZBN, in consistency with the theoretical simulated conformation (Figure 3). The shorter intermolecular contacts in the dimer of $2 \mathrm{PXZBN}$ than $2 \mathrm{PTZBN}$ suggested the latter could better resist aggregation-caused quenching (ACQ).

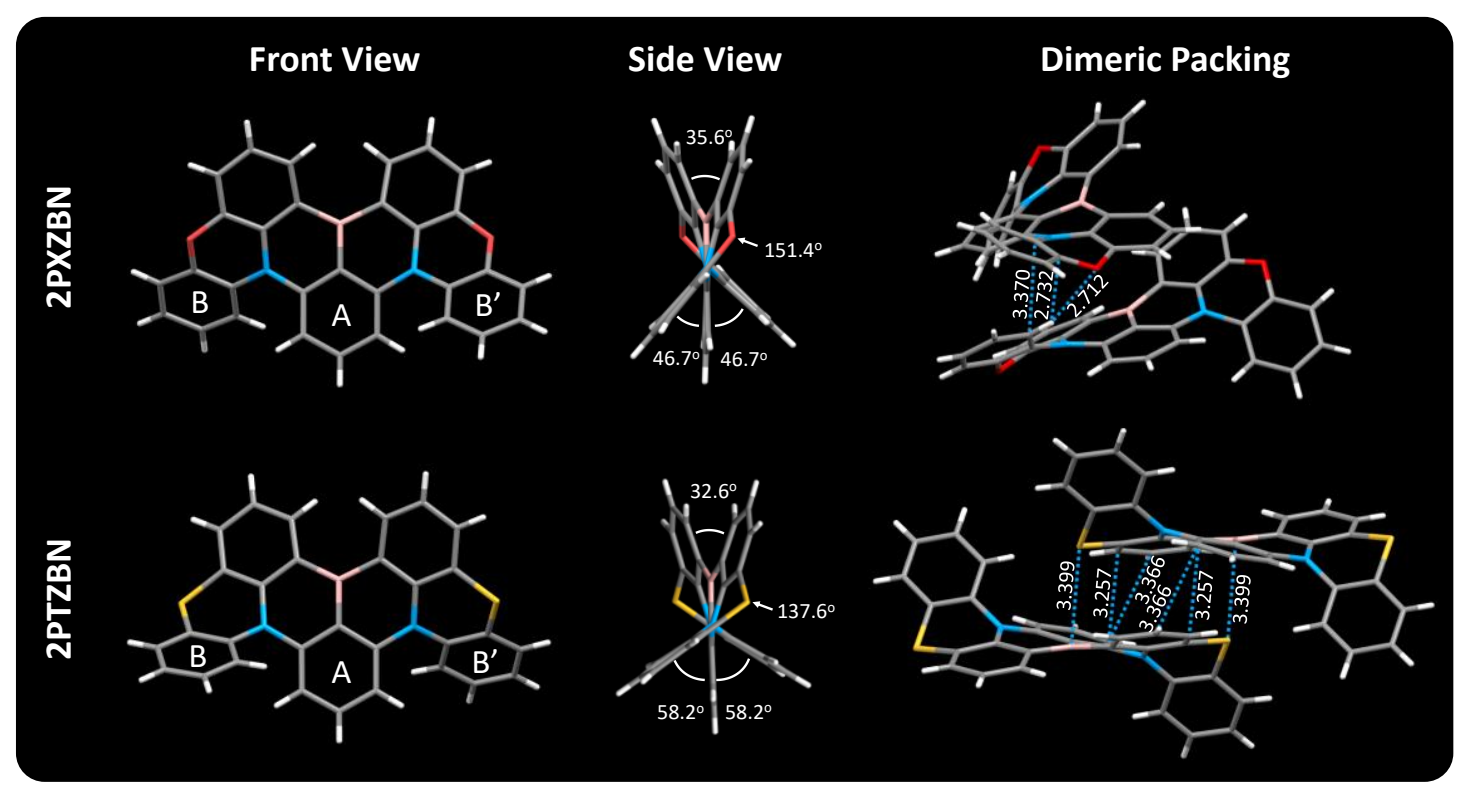

Figure 3. Single crystal structures and packing diagram of 2PXZBN (CCDC: 2063408) and 2PTZBN (CCDC: 2063407). 
The basic photophysical properties of the two emitters were firstly measured in diluted toluene solutions $\left(1 \times 10^{-5} \mathrm{M}\right)$, with their ultraviolet-visible (UV-vis) absorption, fluorescence, and phosphorescence spectra presented in Figure 4. 2PXZBN and 2PTZBN displayed intense absorption bands at 475 and $471 \mathrm{~nm}$, respectively, which could be assigned to their charge-transfer (CT) transitions. The fluorescence spectra of 2PXZBN and 2PTZBN were sharp and structureless, with emission maxima/FWHM of $504 / 34 \mathrm{~nm}$ and 510/39 nm, respectively. The stokes shifts were only $29 \mathrm{~nm}$ and $39 \mathrm{~nm}$ for $2 \mathrm{PXZBN}$ and $2 \mathrm{PTZBN}$, respectively, indicating the small geometry relaxation of their excited states benefited from the rigid molecular structures. The energy levels of the lowest singlet $\left(\mathrm{S}_{1}\right) /$ lowest triplet state $\left(\mathrm{T}_{1}\right)$ were calculated to be $2.60 / 2.41$ and 2.59/2.44 eV from the onset of fluorescence and phosphorescence spectra, yielding a $\Delta E_{\mathrm{ST}}$ of 0.19 and $0.15 \mathrm{eV}$ for 2PXZBN and 2PTZBN, respectively (Figure $4 \mathrm{a}$ and $4 \mathrm{~b}$ ), which were small enough for effective RISC process.

The TADF properties of 2PXZBN and 2PTZBN were subsequently investigated using transient PL measurements in diluted toluene solutions and film states (Figure 4c and 4d). Both compounds exhibited a second-order exponential decay including a nanosecond-scale prompt component $\left(\tau_{\mathrm{PF}}\right)$ and a microsecond-scale delayed component $\left(\tau_{\mathrm{DF}}\right)$ under oxygen-free conditions. The delayed component was almost completely quenched in the presence of oxygen, clearly indicating the contribution of the oxygensensitive triplet excitons and the TADF characteristics of 2PXZBN and 2PTZBN. The $\tau_{\mathrm{PF}} / \tau_{\mathrm{DF}}$ were fitted to be $4.9 \mathrm{~ns} / 11.6 \mu$ s and $4.2 \mathrm{~ns} / 5.0 \mu$ s for $2 \mathrm{PXZBN}$ and $2 \mathrm{PTZBN}$, respectively. Impressively, $2 \mathrm{PTZBN}$ showcased a very short $\tau_{\mathrm{DF}}$ of $5.0 \mu$ s comparable 
to that of the best-performing MR-TADF emitter of $v$-DABNA $(4.1 \mu \mathrm{s})$, implying a fast up-conversion process. The ratio of delayed component was measured to be $16 \%$ for 2PXZBN while it was enhanced to $28 \%$ for $2 \mathrm{PTZBN}$, reflecting a higher emission contribution from the triplet excitons originated from the more efficient RISC process. The photoluminescence quantum yields (PLQYs) were measured to be $77.8 \%$ for 2PXZBN and $58.2 \%$ for 2PTZBN in toluene solutions. The lower PLQY value of 2PTZBN was in line with its lower calculated oscillator strength (Figure 2), which may result from the smaller HOMO-LUMO overlap due to its more twisted molecular configuration (Figure 3). The radiative decay rates $\left(k_{\mathrm{r}, \mathrm{S}}\right)$ of singlet excitons were calculated as $0.93 \times 10^{8} \mathrm{~s}^{-1}$ and $0.79 \times 10^{8} \mathrm{~s}^{-1}$ for $2 \mathrm{PXZBN}$ and $2 \mathrm{PTZBN}$, respectively. Such high $k_{\mathrm{r}, \mathrm{S}}$ values on the order of $10^{8} \mathrm{~s}^{-1}$ are desirable for achieving efficient TADF. The $k_{\text {RISC }}$ values $\left(1.03 \times 10^{5} \mathrm{~s}^{-1}\right.$ and $2.76 \times 10^{5} \mathrm{~s}^{-1}$ for $2 \mathrm{PXZBN}$ and 2PTZBN, respectively) were much higher than most MR-TADF emitters (typically on the order of $10^{3} \sim 10^{4} \mathrm{~s}^{-1}$ ) (see Table S5 in Supporting Information). It should be noted that the $k_{\mathrm{RISC}}$ values of $2 \mathrm{PTZBN}$ were about 2 times faster than those of $2 \mathrm{PXZBN}$, suggesting the larger SOC between the excited states of 2PTZBN involved in the RISC process. Considering the difference in the molecular structures of 2PXZBN and 2PTZBN, the HAE induced by sulfur atoms in $2 \mathrm{PTZBN}$ could play the key role in promoting the intersystem crossing rates of excitons. The photophysical properties of 2PXZBN and 2PTZBN in doped films (mCBP:PO-T2T:emitter $=49.5: 49.5: 1)$ were also measured with similar trends (Figure 4d), and the key data are summarized in Table S4. 
(a)

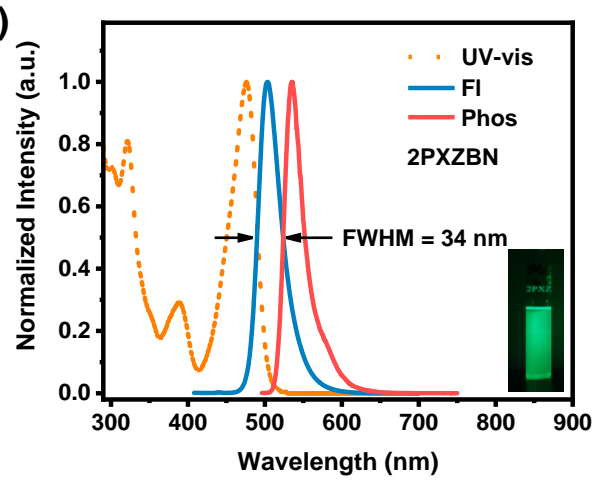

(c)

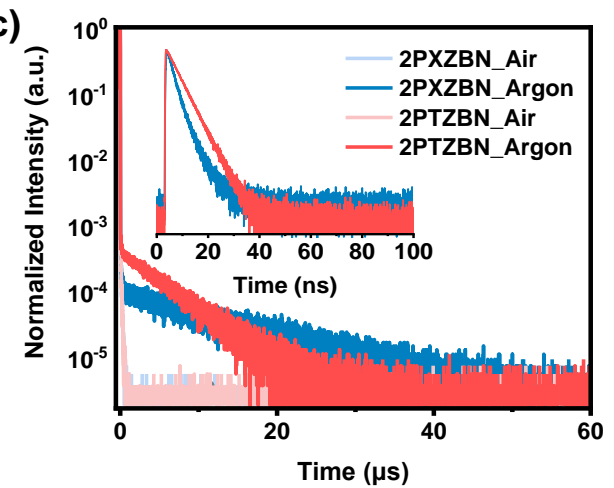

(b)

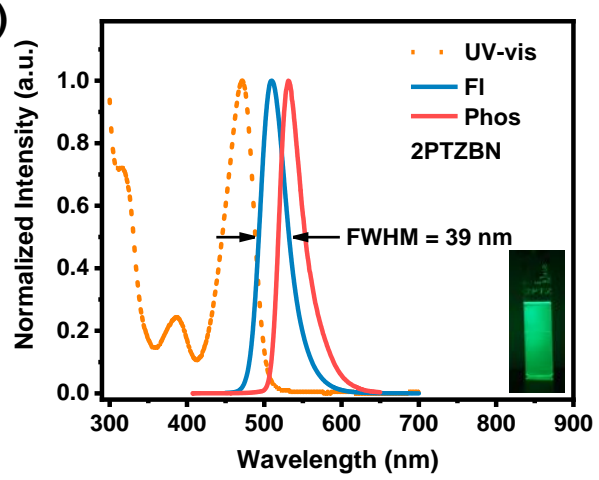

(d)

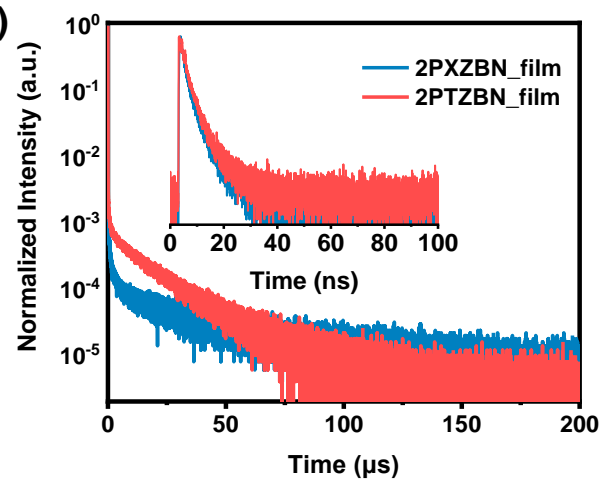

Figure 4. UV-visible absorption (UV-vis), state fluorescence (Fl, measured at $300 \mathrm{~K}$ ) and phosphorescence (Phos, measured at $77 \mathrm{~K}$ ) spectra of 2PXZBN (a) and 2PTZBN (b) in toluene $\left(1 \times 10^{-5} \mathrm{M}\right)$. Transient photoluminescence decay curves of 2 PXZBN and 2PTZBN in toluene $\left(1 \times 10^{-5} \mathrm{M}\right)(\mathrm{c})$ and $1 \mathrm{wt} \% \mathrm{mCBP}: \mathrm{PO}-\mathrm{T} 2 \mathrm{~T}(1: 1)$ doped film (d) under inert atmosphere (Inset: prompt components).

Table 1. Photophysical data of 2PXZBN and 2PTZBN.

\begin{tabular}{|c|c|c|c|c|c|c|c|c|c|c|c|c|}
\hline Emitter & $\begin{array}{c}\lambda_{\mathrm{abs}} \\
{[\mathrm{nm}]}\end{array}$ & $\begin{array}{c}\lambda_{\mathrm{em}} \\
{[\mathrm{nm}]}\end{array}$ & $\begin{array}{c}\text { FWHM } \\
\text { [nm] }\end{array}$ & $\begin{array}{c}\mathrm{HOMO}^{[\mathrm{c}]} \\
{[\mathrm{eV}]}\end{array}$ & $\begin{array}{c}\mathrm{LUMO}^{[\mathrm{d}]} \\
{[\mathrm{eV}]}\end{array}$ & $\begin{array}{l}\mathrm{S}_{1}{ }^{[\mathrm{e}]} \\
{[\mathrm{eV}]}\end{array}$ & $\begin{array}{l}\mathrm{T}_{1}{ }^{[\mathrm{e}]} \\
{[\mathrm{eV}]}\end{array}$ & $\begin{array}{r}\Delta E_{\mathrm{ST}}^{[\mathrm{f}]} \\
{[\mathrm{eV}]}\end{array}$ & $\begin{array}{c}\Phi_{\mathrm{PL}}{ }^{[\mathrm{g}]} \\
{[\%]}\end{array}$ & $\begin{array}{l}\tau_{\mathrm{PF}}^{[\mathrm{h}]} \\
{[\mathrm{ns}]}\end{array}$ & $\begin{array}{c}\tau_{\mathrm{DF}}^{[\mathrm{h}]} \\
{[\mu \mathrm{s}]}\end{array}$ & $\begin{array}{c}k_{\mathrm{RISC}}{ }^{[\mathrm{i}]} \\
{\left[10^{5} \mathrm{~s}^{-1}\right.}\end{array}$ \\
\hline $2 \mathrm{PXZBN}^{[\mathrm{a}]}$ & 475 & 504 & 34 & -5.08 & -2.62 & 2.60 & 2.41 & 0.19 & 78.0 & 4.9 & 11.6 & 1.03 \\
\hline $2 \mathrm{PTZBN}$ & 471 & 510 & 39 & -5.19 & -2.73 & 2.59 & 2.44 & 0.15 & 58.0 & 4.2 & 5.0 & 2.76 \\
\hline $2 \mathrm{PXZBN}^{[\mathrm{b}]}$ & - & 515 & 40 & - & - & - & - & - & 83.7 & 6.8 & 25.3 & 0.56 \\
\hline $2 \mathrm{PTZBN}^{[\mathrm{b}]}$ & - & 519 & 44 & - & - & - & - & - & 80.0 & 4.8 & 16.1 & 1.17 \\
\hline
\end{tabular}

[a] Measured in toluene solutions $\left(10^{-5} \mathrm{M}\right)$. [b] Measured in doped films (mCBP:PO-T2T:emitter = 49.5:49.5:1). [c] Determined from cyclic voltammetry. [d] Determined from HOMO and the energy gap. [e] Lowest excited singlet $\left(\mathrm{S}_{1}\right)$ and triplet $\left(\mathrm{T}_{1}\right)$ energies estimated from the onsets of fluorescence and low-temperature phosphorescence spectra. [f] Energy splitting between the singlet energy and triplet energy. [g] Absolute PLQY. [h] Emission lifetimes for 
prompt fluorescence $\left(\tau_{\mathrm{PF}}\right)$ and delay fluorescence $\left(\tau_{\mathrm{DF}}\right)$. [i] Rate constant of ISC $\left(k_{\mathrm{ISC}}\right)$ and RISC $\left(k_{\mathrm{RISC}}\right)$.

To explore the electroluminescence (EL) performance of 2PXZBN and 2PTZBN as emitters, devices were fabricated with the architecture of indium tin oxide (ITO)/1,4,5,8,9,11-hexaazatriphenylene hexacarbonitrile (HAT-CN) $(5 \mathrm{~nm}) / 1,1$-bis[(di4-tolylamino)phenyl]cyclohexane (TAPC) (30 nm)/1,3-bis(9H-carbazol-9-yl)benzene $(\mathrm{mCP}) \quad(10 \mathrm{~nm}) / \mathrm{EML} \quad(20 \mathrm{~nm}) / \quad$ (1,3,5-triazine-2,4,6-triyl) tris(benzene-3,1-diyl) tris(diphenylphosphine $\quad$ oxide $) \quad($ PO-T2T $) \quad(10 \quad n m) / 1,3,5-\operatorname{tri}(m-$ pyrid-3-ylphenyl)benzene (TmPyPB) (40 $\mathrm{nm}$ )/8-hydroxyquinolinato lithium (Liq) (1.5 $\mathrm{nm}$ )/aluminum (Al) (100 nm). For the emitting layer (EML), mCBP and PO-T2T were used as the exciplex hosts, in which mCBP was the electron donor and PO-T2T acted as the electron acceptor. Here, the use of such mixed hosts can not only balance carrier transport in EML, but also lower the energy injecting barrier for both holes and electrons. The ratio of mCBP:PO-T2T:emitter in the EML was set as 49.5:49.5:1. The alignment of energy levels of the devices, current density-voltage-luminescence $(J-V$ $L)$ curves, current efficiency-luminance-power efficiency $(C E-L-P E)$ curves, external quantum efficiency-luminance $(E Q E-L)$ curves, and EL spectra for the devices were detailed in Figure 5, and the key device parameters were summarized in Table 2.

The two devices exhibited a low turn-on voltage $\left(V_{\text {on }}\right)$ of $3.0 \mathrm{~V}$ (recorded at a brightness of $1 \mathrm{~cd} \mathrm{~m}^{-2}$ ), attributed to the suitable energy level alignment of the functional materials. The emission maxima for the devices based on 2PXZBN and 2PTZBN are $522 \mathrm{~nm}$ and $527 \mathrm{~nm}$, with Commission Internationale de l'Eclairage (CIE) coordinates of $(0.28,0.64)$ and $(0.28,0.65)$, FWHM values of 60 and $58 \mathrm{~nm}$, 
respectively. The EL spectra of the two emitters exhibited a redshift of approximately $10 \mathrm{~nm}$ compared with the emission in doped films, possibly attributing to the optical cavity effect in the device. ${ }^{44,45}$ Despite their similar EL spectra, 2PXZBN and 2PTZBN exhibited quite significant differences in their device efficiencies. The maximum PE $\left(\mathrm{PE}_{\max }\right), \mathrm{CE}\left(\mathrm{CE}_{\max }\right)$, and $\mathrm{EQE}\left(\mathrm{EQE}_{\max }\right)$ of $2 \mathrm{PXZBN}$ based device were $58.3 \mathrm{~lm} \mathrm{~W} \mathrm{~W}^{-1}$, $64.9 \mathrm{~cd} \mathrm{~A}^{-1}$, and $17.7 \%$, respectively, while those of 2PTZBN based device were 86.6 $\operatorname{lm~} \mathrm{W}^{-1}, 96.5 \mathrm{~cd} \mathrm{~A} \mathrm{~A}^{-1}$, and $25.5 \%$. The 2PTZBN-based device manifested $44 \%$ enhancement of $\mathrm{EQE}_{\max }$ compared to one based on $2 \mathrm{PXZBN}$, representing one of the highest EQE values among the green MR-TADF OLEDs. Importantly, at the luminance of $100 \mathrm{~cd} \mathrm{~m}^{-2}$, the EQEs of 2PXZBN and 2PTZBN based devices were $15.3 \%$ and $21.7 \%$, respectively, both corresponding to a moderate roll-off of $14 \%$. However, at the luminance of $1000 \mathrm{~cd} \mathrm{~m}^{-2}$, the roll-off value was $33 \%$ for $2 \mathrm{PTZBN}$, while the value was almost doubled in the case of 2PXZBN, reaching 58\%. The significantly alleviated EQE roll-off in the case of 2PTZBN implied the effectively suppressed exciton quenching in the device. The excellent device performances of 2PTZBN most probably benefited from its shorter delayed lifetime and higher RISC rate, which resulted in fast and efficient upconversion of triplet excitons to avoid the triplet-related quenching effects. It should be recalled that 2PTZBN exhibited a lower PLQY and $k_{\mathrm{r}, \mathrm{S}}$, while the

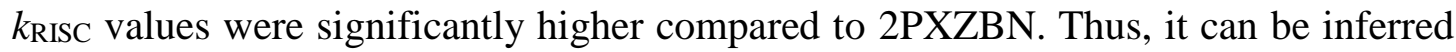
that the efficient RISC process benefiting from the HAE of sulfur atoms could elevate the device performance. 
(a)

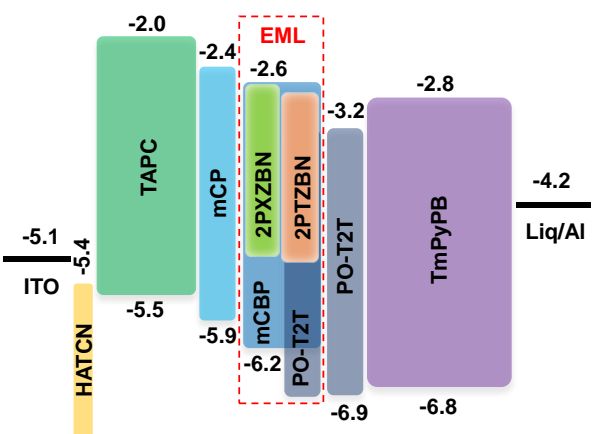

(c)

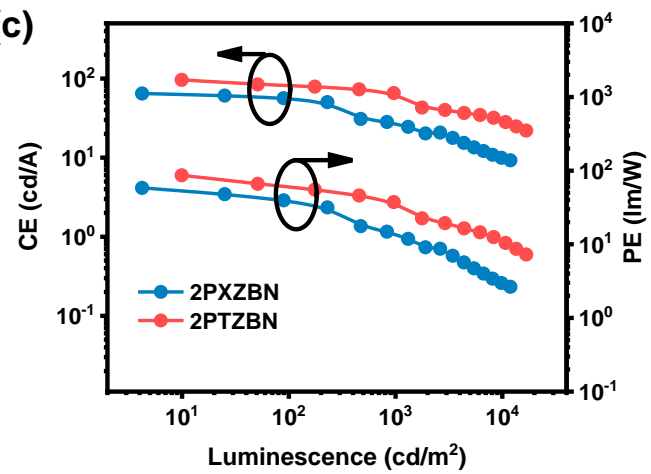

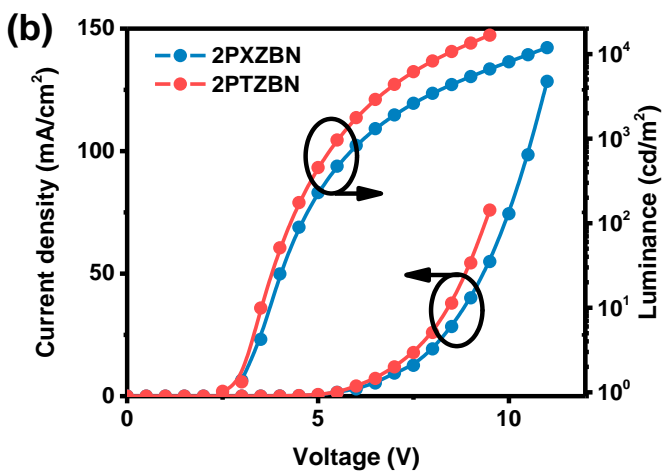

(d)

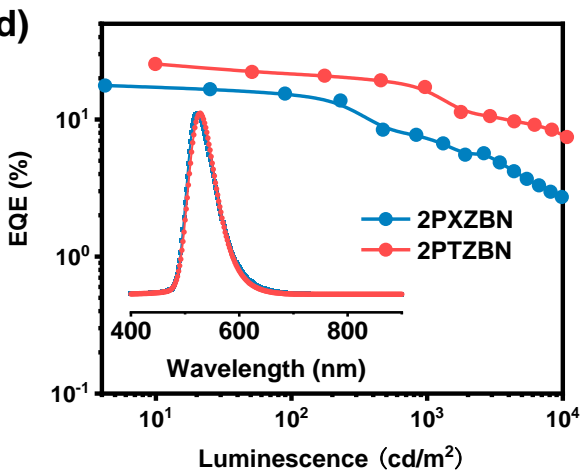

Figure 5. (a) Device architecture for the MR-TADF OLEDs with energy level alignment of the relevant materials; (b) current density and luminance versus voltage $(J-V-L)$ characteristics; (c) current efficiency (CE) and power efficiency (PE) versus luminance; (d) external quantum efficiency (EQE)-luminance curves with normalized electroluminescence (EL) spectra as the inset.

Table 2. Summary of the electroluminescent data.

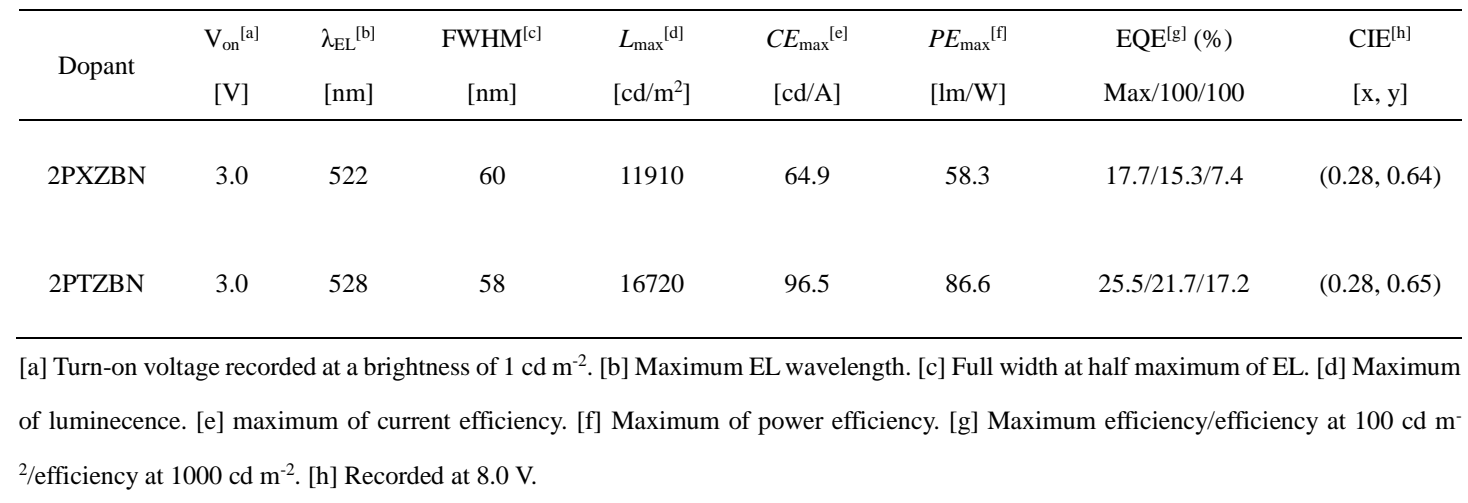

In conclusion, we developed two new B-N based MR-TADF emitters by 
incorporating sulfur (or oxygen) atom into the B-N polycyclic framework. The theoretical calculations and photophysical studies revealed that the sulfur-based MRTADF emitter (2PTZBN) enable larger SOC and faster $k_{\text {RISC }}$ values in comparison with the oxygen-based counterpart (2PXZBN). Significant boost of EQEs from $17.7 \%$ to $25.5 \%$ and better resistance to roll-off were achieved in the OLEDs when switching the emitter from 2PXZBN to 2PTZBN. We believe the new strategy to promote MR-TADF by heavy-atom effect would shed light on the development of high-performance MRTADF emitters and devices.

\section{Conflicts of interest}

The authors declare no conflict of interest.

\section{Acknowledgements}

This research is financially supported by the National Natural Science Foundation of China (21805195 and 91833304), the Shenzhen Science and Technology Program (KQTD20170330110107046 and JCYJ20190808151209557). We thank the Instrumental Analysis Center of Shenzhen University for analytical support.

\section{References}

1. T. Hatakeyama, K. Shiren, K. Nakajima, S. Nomura, S. Nakatsuka, K. Kinoshita, J. Ni, Y. Ono and T. Ikuta, Adv. Mater., 2016, 28, 2777-2781.

2. H. Jiang, Y. Cao, Q. Yang, L. Xian, Y. Tao, R. Chen and W. Huang, J. Phys. Chem. Lett., 2020, 11, 7739-7754.

3. Z. Huang, S. Wang, R. D. Dewhurst, N. V. Ignat'ev, M. Finze and H. Braunschweig, Angew. Chem. Int. Ed., 2019, 59, 8800-8816. 
4. S. S. Kothavale and J. Y. Lee, Adv. Optical Mater, 2020, 8, 2000922.

5. H. Lee, D. Karthik, R. Lampande, J. H. Ryu and J. H. Kwon, Front Chem., 2020, 8, 373 .

6. S. Madayanad Suresh, D. Hall, D. Beljonne, Y. Olivier and E. Zysman-Colman, Adv. Funct. Mater., 2020, 30, 1908677.

7. J.-M. Teng, Y.-F. Wang and C.-F. Chen, J. Mater. Chem. C, 2020, 8, 1134011353.

8. X. Liang, Z. P. Yan, H. B. Han, Z. G. Wu, Y. X. Zheng, H. Meng, J. L. Zuo and W. Huang, Angew. Chem. Int. Ed., 2018, 57, 11316-11320.

9. J. Knoeller, G. Meng, X. Wang, D. Hall, A. Pershin, D. Beljonne, Y. Olivier, S. Laschat, E. Zysman-Colman and S. Wang, Angew. Chem. Int. Ed., 2019, 59, 3156-3160.

10. X. Li, Y. Z. Shi, K. Wang, M. Zhang, C. J. Zheng, D. M. Sun, G. L. Dai, X. C. Fan, D. Q. Wang, W. Liu, Y. Q. Li, J. Yu, X. M. Ou, C. Adachi and X. H. Zhang, ACS Appl. Mater. Interfaces, 2019, 11, 13472-13480.

11. S. Oda, B. Kawakami, R. Kawasumi, R. Okita and T. Hatakeyama, Org. Lett., 2019, 21, 9311-9314.

12. Y. Yuan, X. Tang, X. Y. Du, Y. Hu, Y. J. Yu, Z. Q. Jiang, L. S. Liao and S. T. Lee, Adv. Optical Mater., 2019, 7, 1801536.

13. N. Ikeda, S. Oda, R. Matsumoto, M. Yoshioka, D. Fukushima, K. Yoshiura, N. Yasuda and T. Hatakeyama, Adv. Mater., 2020, 32, 2004072.

14. S. Oda, T. Hatakeyama, W. Kumano, T. Hama, R. Kawasumi and K. Yoshiura, Angew. Chem. Int. Ed., 2020, 60, 2882-2886.

15. X. Qiu, G. Tian, C. Lin, Y. Pan, X. Ye, B. Wang, D. Ma, D. Hu, Y. Luo and Y. Ma, Adv. Optical Mater., 2020, 8, 202001845.

16. Y. Xu, Z. Cheng, Z. Li, B. Liang, J. Wang, J. Wei, Z. Zhang and Y. Wang, Adv. Optical Mater., 2020, 8, 1902142.

17. Y. Zhang, D. Zhang, J. Wei, X. Hong, Y. Lu, D. Hu, G. Li, Z. Liu, Y. Chen and L. Duan, Angew. Chem. Int. Ed., 2020, 60, 17499-17503.

18. S. N. Zou, C. C. Peng, S. Y. Yang, Y. K. Qu, Y. J. Yu, X. Chen, Z. Q. Jiang and L. S. Liao, Org. Lett., 2021, 23, 958-962.

19. C.-Y. Chan, M. Tanaka, Y.-T. Lee, Y.-W. Wong, H. Nakanotani, T. Hatakeyama and C. Adachi, Nat. Photonics, 2021, DOI: 10.1038/s41566-020-00745-Z.

20. H. Min, I. S. Park and T. Yasuda, Angew. Chem. Int. Ed., 2021, DOI: 10.1002/anie.202016914.

21. S. Xu, Q. Yang, Y. Zhang, H. Li, Q. Xue, G. Xie, M. Gu, J. Jin, L. Huang and R. Chen, Chinese Chem. Lett., 2020, DOI: 10.1016/j.cclet.2020.10.022.

22. Soon Ok Jeon, Kyung Hyung Lee, Jong Soo Kim, Soo-Ghang Ihn, Yeon Sook Chung, Ji Whan Kim, Hasup Lee, Sunghan Kim, Hyeonho Choi and Jun Yeob Lee, Yeon Sook Chung, Nat. Photonics, 2021, DOI: 10.1038/s41566-02100763-5.

23. H. Uoyama, K. Goushi, K. Shizu, H. Nomura and C. Adachi, Nature, 2012, 492, 234-238.

24. F. B. Dias, T. J. Penfold and A. P. Monkman, Methods Appl. Fluoresc., 2017, 5, 
012001.

25. M. Y. Wong and E. Zysman-Colman, Adv. Mater., 2017, 29. 1605444.

26. Z. Yang, Z. Mao, Z. Xie, Y. Zhang, S. Liu, J. Zhao, J. Xu, Z. Chi and M. P. Aldred, Chem. Soc. Rev., 2017, 46, 915-1016.

27. Y. Liu, C. Li, Z. Ren, S. Yan and M. R. Bryce, Nat. Rev. Mater., 2018, 3, 18020.

28. D. Wang, C. Cheng, T. Tsuboi and Q. Zhang, CCS Chem., 2020, 2, 1278-1296.

29. S. H. Han, J. H. Jeong, J. W. Yoo and J. Y. Lee, J. Mater. Chem. C, 2019, 7, 3082-3089.

30. Y. Kondo, K. Yoshiura, S. Kitera, H. Nishi, S. Oda, H. Gotoh, Y. Sasada, M. Yanai and T. Hatakeyama, Nat. Photonics, 2019, 13, 678-682.

31. Y. Zhang, D. Zhang, J. Wei, Z. Liu, Y. Lu and L. Duan, Angew. Chem. Int. Ed., 2019, 58, 16912-16917.

32. Y. Xu, C. Li, Z. Li, Q. Wang, X. Cai, J. Wei and Y. Wang, Angew. Chem. Int. Ed., 2020, 59, 17442-17446.

33. M. Yang, I. S. Park and T. Yasuda, J. Am. Chem. Soc., 2020, 142, 19468-19472.

34. D. Zhang, X. Song, A. J. Gillett, B. H. Drummond, S. T. E. Jones, G. Li, H. He, M. Cai, D. Credgington and L. Duan, Adv. Mater., 2020, 32, 1908355.

35. P. K. Samanta, D. Kim, V. Coropceanu and J.-L. Bredas, J. Am. Chem. Soc., 2017, 139, 4042-4051.

36. F. Chen, L. Zhao, X. Wang, Q. Yang, W. Li, H. Tian, S. Shao, L. Wang, X. Jing and F. Wang, Sci. China Chem., 2021, DOI: 10.1007/s11426-020-9944-1.

37. T. Agou, K. Matsuo, R. Kawano, I. S. Park, T. Hosoya, H. Fukumoto, T. Kubota, Y. Mizuhata, N. Tokitoh and T. Yasuda, ACS Materials Lett., 2020, 2, 28-34.

38. J. Xu, X. Zhu, J. Guo, J. Fan, J. Zeng, S. Chen, Z. Zhao and B. Z. Tang, ACS Materials Lett., 2019, 1, 613-619.

39. A. Kretzschmar, C. Patze, S. T. Schwaebel and U. H. Bunz, J. Org. Chem., 2015, 80, 9126-9131.

40. Y. Xiang, Y. Zhao, N. Xu, S. Gong, F. Ni, K. Wu, J. Luo, G. Xie, Z.-H. Lu and C. Yang, J. Mater. Chem. C, 2017, 5, 12204-12210.

41. W. Zhang, J. Jin, Z. Huang, S. Zhuang and L. Wang, Sci. Rep., 2016, 6, 30178.

42. M. Einzinger, T. Zhu, P. de Silva, C. Belger, T. M. Swager, T. Van Voorhis and M. A. Baldo, Adv Mater, 2017, 29. 1701987.

43. S. Gan, S. Hu, X. L. Li, J. Zeng, D. Zhang, T. Huang, W. Luo, Z. Zhao, L. Duan, S. J. Su and B. Z. Tang, ACS Appl. Mater. Interfaces, 2018, 10, 17327-17334.

44. J. Lee, N. Aizawa and T. Yasuda, J. Mater. Chem. C, 2018, 6, 3578-3583.

45. L. Cao, K. Klimes, Y. Ji, T. Fleetham and J. Li, Nat. Photonics, 2020, DOI: 10.1038/s41566-020-00734-2. 